\title{
Mean Platelet Volume in Euthyroid Patients with Hashimoto's Thyroiditis
}

\begin{abstract}
Dr. Ayse Carlioglu ${ }^{1}$, Dr.Ozge Timur ${ }^{2}$, Dr.Senay Arikan Durmaz ${ }^{2}$, Dr. Mehmet Emin Ayhan ${ }^{1}$
Erzurum Regional Training and Research Hospital, Department of Endocrinology ${ }^{1}$, Erzurum, TURKEY

Erzurum Regional Training and Research Hospital, Department of Internal Medicine ${ }^{2}$, Erzurum, TURKEY

INTRODUCTION AND OBJECTIVE:
\end{abstract}

Hashimoto's thyroiditis (HT) is an autoimmune disease in which a breakdown of immune tolerance is caused by interplay of a variety of immunologic, genetic, and environmental factors. Mean platelet volume (MPV) is the measure of platelet size. MPV possibly is a simple way to estimate platelet activity. Activated platelets play an important role in the pathogenesis of vascular disease especially coronary heart diseases. Larger platelets are metabolically and enzymatically more active and have greater protrombotic potential.In this study we aimed to investigate MPV levels in euthyroid Hashimoto's thyroiditis patients.

\section{MATERIAL AND METHODS:}

Fifty-one euthyroid patients with Hashimoto's thyroiditis attending to our outpatient clinic of endocrinology department and 51 age and body mass index matched healty subjects were included this study. All patients with HT were euthyroid state. Anti-thyroid peroxidase (antiTPO) antibody and anti-tiroglobulin (Anti-Tg) antibody were positive. All the study subjects were evaluated by biochemical and platelet parameters. Student's $t$-test was done to find the significance of difference between means when ever applicable. One tailed Pearson' $\mathrm{s}$ correlation test was done to find the correlation between various variables. Linear regression analysis was done whenever appropriate. Chi square test, Chi square with Yates correction and Fisher's exact test, wherever applicable was done to test the association between two findings.

\section{RESULTS:}

There were no significant differences in age, sex and body mass index between study and control groups. Laboratory parameters are shown in Table 2. Anti- TPO and tiroglobulin levels were significantly higher in study group. ( $p$-anti-TPO 0.00, p-tg 0,09) There was no significant difference in FT3, FT4 and TSH levels between two groups. MPV levels were significantly higher in Hashimato's disease group $(p=0.00$, Figure I). There was a positive correlation between anti-TPO and MPV levels $(p=0,042)$. Positive correlation was also observed between anti tg and MPV levels $(p=0,033)$.

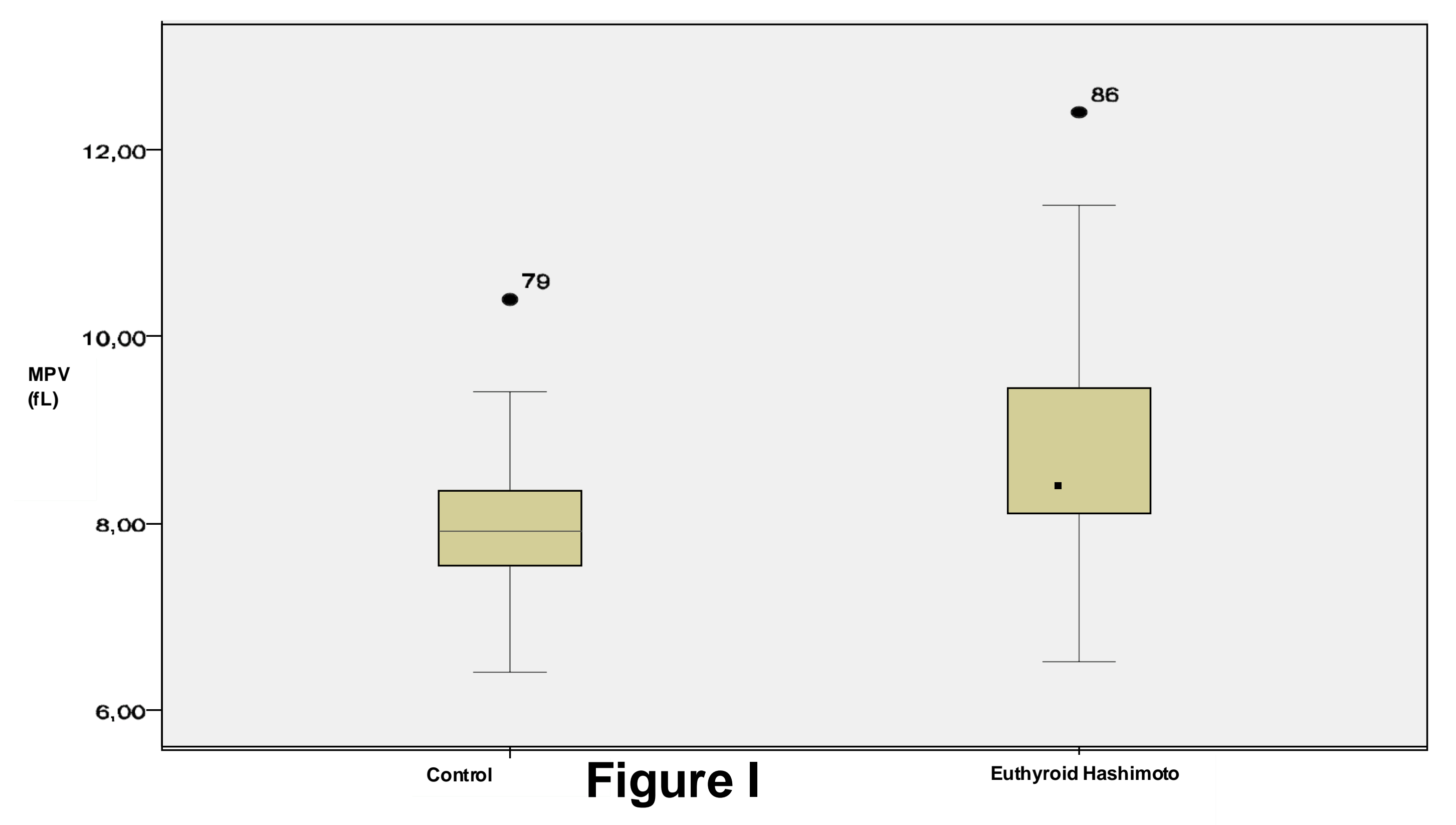

\section{DISCUSSION:}

This study demonstrate that MPV levels in Hashimoto's patients tend to be higher than healty controls even if in euthyroid state. Since platelets play a crucial role in the pathogenesis of thrombotic diseases, it had been proposed that increased MPV might be a cardiovascular risk factor. Endler et al. reported that regardless of the extent of the coronary lesions among patients with coronary artery disease, those with higher MPV values had been found to have a greater risk of acute myocardial infarction than those with lower MPV. Previous studies reported that there is a strong relationship between cardiovasculer system and thyroid hormones. In a recent study Ylmaz et al. reported that increased MPV levels in subclinical hypothyroid patients are releated with increased cardiovasculer complication risk and patients have stil higher MPV values even though they become euthyroid after levothyroxine therapy. In our study all the patients have higher anti-TPO and anti-tg levels than control group. There is also positive correlation between anti-TPO, anti-tg levels and MPV levels Unlike other studies all of our patients were in eythyroid state and recevie no levothyroxine therapy. This data shows that even if euthyroid Hashimoto' $s$ patients MPV levels are higher than control group. This is the first study to evaluate the MPV levels in euthyroid autoimmune thyroid disesase. As a result autoimmune thyroid disease even in euthyroid state is associated with higher MPV levels.

\section{CONCLUSION:}

Our results suggest that patients with autoimmune thyroid disesases have higher MPV levels even if in euthyroid state than healty controls. As higher MPV levels are close related with cardiovasculer diseases EH patients have greater risk of atherothrombotic complications than controls. 